\title{
The corallivorous flatworm Amakusaplana acroporae: an invasive species threat to coral reefs?
}

\author{
Benjamin C. C. Hume • Cecilia D'Angelo • \\ Anna Cunnington $\cdot$ Edward G. Smith . \\ Jörg Wiedenmann
}

Received: 25 June 2013/ Accepted: 4 November 2013/Published online: 20 November 2013

(c) The Author(s) 2013. This article is published with open access at Springerlink.com

\begin{abstract}
Fatal infestations of land-based Acropora cultures with so-called Acropora-eating flatworms (AEFWs) are a global phenomenon. We evaluate the hypothesis that AEFWs represent a risk to coral reefs by studying the biology and the invasive potential of an AEFW strain from the UK. Molecular analyses identified this strain as Amakusaplana acroporae, a new species described from two US aquaria and one natural location in Australia. Our molecular data together with life history strategies described here suggest that this species accounts for most reported cases of AEFW infestations. We show that local parasitic activity impairs the light-acclimation capacity of the whole host colony. A. acroporae acquires excellent camouflage by harbouring photosynthetically competent, host-derived zooxanthellae and pigments of the greenfluorescent protein family. It shows a preference for Acropora valida but accepts a broad host range. Parasite survival in isolation (5-7 d) potentially allows for an invasion when introduced as non-native species in coral reefs.
\end{abstract}

Communicated by Biology Editor Dr. Anastazia Banaszak

Electronic supplementary material The online version of this article (doi:10.1007/s00338-013-1101-6) contains supplementary material, which is available to authorized users.

B. C. C. Hume - C. D'Angelo - A. Cunnington .

E. G. Smith · J. Wiedenmann $(\bowtie)$

Ocean and Earth Science, University of Southampton, National Oceanography Centre Southampton (NOCS), European Way, Southampton SO143ZH, UK

e-mail: joerg.wiedenmann@noc.soton.ac.uk

Present Address:

E. G. Smith

Centre for Genomics and Systems Biology, New York

University, Abu Dhabi, PO Box 129 188, Abu Dhabi, UAE
Keywords Acropora-eating flatworms .

Amakusaplana acroporae - Invasive species - GFP-

like fluorescent proteins - Corallivory ·

Photoprotection - Green fluorescent protein .

Biomarker

\section{Introduction}

Coral reefs, one of the most biodiverse and productive ecosystems in the world, are sensitive to a range of perturbations including the potentially devastating effects of corallivory, defined as the direct assimilation of live coral tissue (Hughes et al. 2007; Rotjan and Lewis 2008).

Fatal infestations of aquarium-cultured acroporid corals with corallivorous flatworms/AEFWs have been globally reported (Electronic Supplemental Material, ESM Table 1, Fig. 1). Early stages of infestations with these well-camouflaged AEFWs often manifest as pale 'bite marks' (Nosratpour 2008; ESM Table 1). Most recently AEFWs from two US aquaria were described as a new species (Amakusaplana acroporae) within the phylum Platyhelminthes (Rawlinson et al. 2011), which was subsequently found on A. valida in one natural location off Lizard Island, Great Barrier Reef (GBR) (Rawlinson and Stella 2012). The guts and parenchyma of A. acroporae contained nematocysts and zooxanthellae, suggesting that the damage to the corals is due to feeding by the worms (Rawlinson et al. 2011; Rawlinson and Stella 2012). Another Acropora-associated worm is an acoel from the phylogenetically distant taxon Waminoa (phylum Acoelomorpha), which shows a striking phenotypic similarity to A. acroporae (Matsushima et al. 2010), making it difficult to assign the reported AEFW infestation to one or the other species. Also, other representatives of Waminoa live epizoic on corals; however, there are no reports on them 
being corallivores, and their zooxanthellae are not derived from corals (Barneah et al. 2007). They are thought to consume only coral mucus but may harm the coral by shading and related negative effects on the coral's photophysiology when occurring in high numbers (Barneah et al. 2007; Haapkylä et al. 2009; Naumann et al. 2010). Although A. acroporae has been found on the GBR, the natural origin and biogeographic range of the aquarium strains of AEFWs are unclear and no natural predators are known, making it difficult to control infestations in land-based Acropora cultures. Reports of aquaria strains of AEFWs include regions close to natural coral reefs such as Florida, Thailand or Hong Kong (Fig. 1), raising the question whether AEFWs represent a risk for natural coral communities if released in the environment. The introduction of non-native species in natural ecosystems including coral reefs may have dramatic consequences, and a significant number of ornamental species have become invasive in aquatic ecosystems (Padilla and Williams 2004). The invasion, for instance, of the Mediterranean Sea by the macroalga Caulerpa taxifolia (Wiedenmann et al. 2001) or the Caribbean Sea by the lionfishes Pterois sp. (Betancur-R et al. 2011) was triggered by the release of aquarium strains.

To judge the danger that AEFWs represent as potential invasive species for natural coral reefs, we studied the biology of an AEFW strain obtained from the ornamental trade in the UK. We applied molecular taxonomy approaches to identify the species, analysed host preference, feeding behaviour, association with zooxanthellae, effects on the host physiology and survival times in the absence of hosts.

\section{Methods}

Culture and aquarium experiments

An AEFW strain acquired from a local ornamental trader in Southampton (UK) was co-cultured for 6 months with a diverse range of acroporids and numerous other coral species (ESM Table 2) in a separated compartment of the experimental coral mesocosm of the Coral Reef Laboratory at the National Oceanography Centre Southampton (D'Angelo and Wiedenmann 2012). Host preference was judged using a classification scheme shown in ESM Table 2. For subsequent studies, AEFWs were dislodged from the host by a seawater jet. Survival in the absence of host corals was evaluated by maintaining ten isolated specimens in a partially shaded compartment without access to corals. To test the response of the AEFWs to increased temperatures, infested fragments of Acropora sp. were subjected to a heat stress treatment as described in (Wiedenmann et al. 2013). Horizontally growing Acropora millepora replicate colonies (infested and non-infested) were turned by $180^{\circ}$, and light acclimation was monitored through the fluorescence increase in the newly light exposed branch surface (D'Angelo et al. 2008).

\section{Molecular identification of AEFW and zooxanthellae}

Genomic DNA (gDNA) was prepared from a pool of 6 AEFWs collected from an A. millepora colony, the infested host colony itself and a co-cultured Acropora microphthalma colony using a protocol described in (Hume et al. 2013).

Phylotyping of zooxanthellae (Symbiodinium spp.) in the corals and in the AEFW was conducted by amplifying, cloning and sequencing of the ITS1-5.8S-ITS2 ribosomal DNA using zooxanthellae-specific primers (ESM Table 3) (Hume et al. 2013; Savage et al. 2002). Sequences were deposited in Genbank (accession numbers JN711475-JN711498).

The 18S rDNA region of the AEFW genomic DNA was amplified as two overlapping fragments, using primers designed against conserved regions of polyclad $18 \mathrm{~S}$ sequences (ESM Table 3). A primer pair to amplify a 618-bp fragment of the $28 \mathrm{~S}$ region was developed using polyclad 28S sequences available in GenBank (ESM Table 3). Sequences were deposited in Genbank (accession numbers JN711499-JN711500).
Fig. 1 Global distribution of AEFW infestations of landbased coral cultures reported on the internet (numbers 1-16) and in scientific literature (letters A$D$ ). Locations in proximity to coral reefs are highlighted by boxes in bold style. Black spots show the distribution of tropical coral reefs. The letter $(E)$ marks the location at which A, acroporae was found in the wild (Rawlinson and Stella 2012)

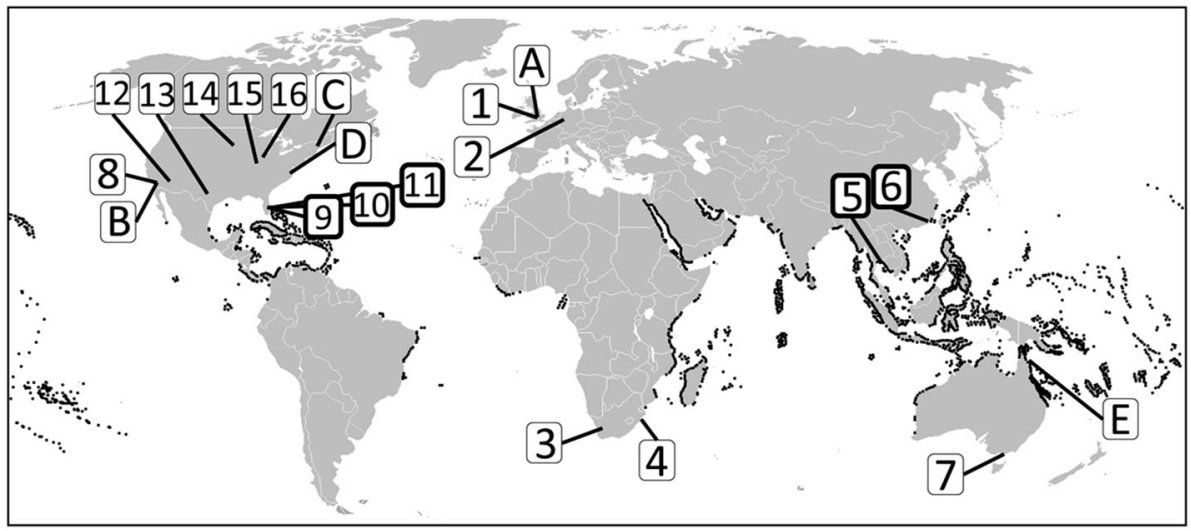



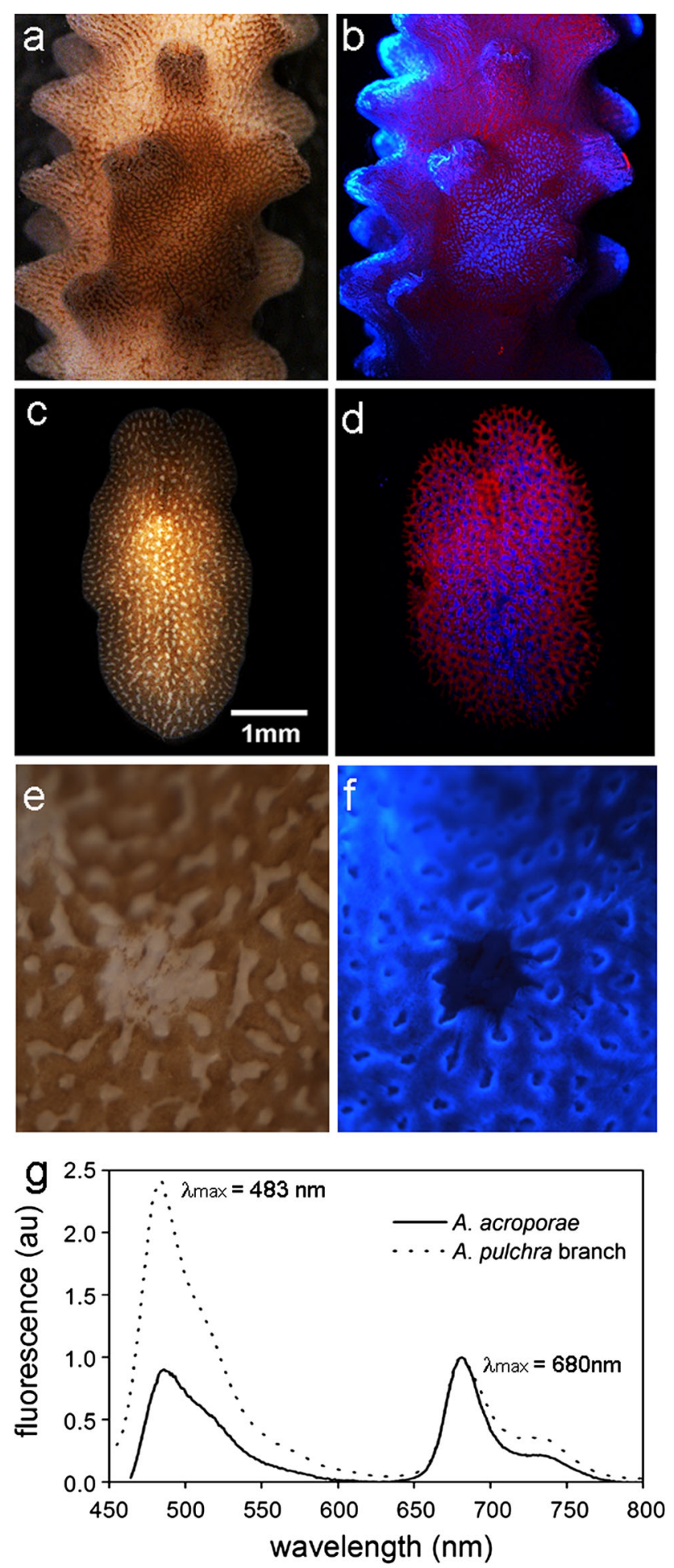

Phylogenetic analysis of 18S and ITS2 sequences was performed using MEGA 5 (Tamura et al. 2011). Sequences were aligned using ClustalW, and phylogenetic trees were constructed using maximum likelihood (ML) methods. Analysis was performed to infer an optimal nucleotide substitution method. For $18 \mathrm{~S}$ analysis, a Tamura-Nei with
Fig. 2 Acquired camouflage strategy of the AEFW. a, b Micrograph of A. pulchra with a representative AEFW of the UK aquarium strain attached. c, d Images of the isolated parasite. e, f Close-up photographs of a typical 'bite mark' left by the parasite in the tissue of the branch underside of A. pulchra. Photographs were acquired under the microscope under white light conditions $(\mathbf{a}, \mathbf{c}, \mathbf{e})$ and in the fluorescence mode using a CFP/dsRed filter set (b, d, f). Chlorophyll fluorescence shows in red; the fluorescence of the cyan apulFP583 appears in blue. $\mathrm{g}$ Fluorescence emission spectra $\left(\lambda_{\mathrm{exc}}=420 \mathrm{~nm}\right)$ of the host coral tissue (A. pulchra, dotted line) and of the isolated parasite (solid line)

gamma distribution and 5 rate categories were selected based on Akaike Information Criterion. ITS2 sequences were analysed using the Hasegawa-Kishino-Yano model with equal rates. The certainty of ML nodes was tested with bootstrap analysis (100 replications).

Photographic documentation, fluorescence measurements and microscopy

Coral fluorescence was imaged using a yellow longpass filter and a $\sim 450-n m$ excitation light source (Nightsea, Andover, USA). Microscopic close-ups were obtained as described in (D'Angelo et al. 2012; Smith et al. 2013).

Photosynthesis efficiency of zooxanthellae was determined by pulse amplitude modification (PAM) fluorometry using a Diving-PAM (Walz) for a pool of 5 AEFW specimens.

Fluorescence spectra were recorded using a Varian Cary Eclipse fluorescence spectrometer (Varian, Palo Alto, USA) (D’Angelo et al. 2008, 2012).

\section{Results and discussion}

After co-culturing the AEFW strain with a diverse range of acroporids and fifteen other scleractinian coral species for 6 months in a separated compartment of our experimental coral mesocosm (D'Angelo and Wiedenmann 2012), the AEFWs were still exclusively found on acroporid corals (ESM Table 2). The highest to lowest host preference was as followed: A. valida $>A$. millepora $>A$. pulchra $>$ A. polystoma $>$ A. yongei $>$ A. gemmifera $>$ A. microphthalma $>$ A. tortuosa. The AEFWs, reaching a size of up to $\sim 6 \mathrm{~mm}$, were always found attached to the shaded branch sides. Microscopical inspection revealed the excellent camouflage of the AEFWs that mimics both the colour and pigment distribution of the host (Fig. 2a, c; ESM Fig. 1a). Due to their camouflage, the AEFWs could be easily overlooked, but their previously described characteristic pale 'bite marks' on the branch underside (ESM Table 1) turned out to be reliable indicators of an infestation (Fig. 2e, ESM Fig. 1a). As described by Rawlinson et al. (2011), another clue to the presence of the parasite was 
clusters of $\sim 5$ to $\sim 90$ eggs found only on bare skeleton with close proximity to live coral tissue (ESM Fig. 1b-c). The parasites appear to be rather stationary, and a single individual was observed for 5 weeks on a $\sim 5 \mathrm{~cm}$ replicate colony of A. millepora before it was removed for experimental purposes. The activity of this specimen, restricted to the underside of the branch, had a significant effect on the overall physiology of the host coral, essentially preventing it to acclimatise to higher light levels (D'Angelo et al. 2008; Smith et al. 2013) by increasing the accumulation of GFP-like proteins in the light exposed branch side (Fig. 3a). Accordingly, infested colonies were less fluorescent as compared to healthy counterparts (Fig. 3b, c; ESM Fig. 2). Hence, host fluorescence can be used as a further indicator of the presence of the parasites, underlining the potential of GFP-like proteins to serve as stress indicators for corals (D'Angelo et al. 2012).

Interestingly, the AEFWs collected from A. pulchra show fluorescence patterns perfectly matching those of the host, with the coral fluorescence being derived from the cyan GFP-like protein apulFP583 (D'Angelo et al. 2008) and red chlorophyll fluorescence of zooxanthellae (Oswald et al. 2007) (Fig. 2). In contrast, depending on their developmental stage, eggs and embryos show only a weak blue or orange fluorescence under UV (365 nm) excitation (ESM Fig. 1c). The absence of fluorescence in the feeding marks in the coral tissues proposes that the parasites extract the fluorescent pigments from the host (Fig. 2f). The cyan fluorescent pigments appear to be evenly distributed in the parenchyma of the parasites, suggesting an incorporation of host pigments in their functional form to perfect the camouflage of the parasite (Fig. 2b, d). Such strategy could be facilitated by the high stability and slow turnover of coral GFP-like proteins (Leutenegger et al. 2007).

Molecular analysis proved that the algal cells in the AEFWs from an A. millepora colony are zooxanthellae. The distribution of Symbiodinium clades in the AEFW closely matched the host coral's algal community and was dissimilar to the one of co-cultured A. microphtalma (Fig. 4a), providing evidence that the parasites acquire zooxanthellae from the host by feeding. This is further supported by the lack of zooxanthellae in the feeding marks (Fig. 2e; ESM Fig. 1a). A heat-stressed AEFW individual was observed to release most of its zooxanthellae within a few seconds after contracting movements (presumably via the pharynx), demonstrating that at least most of the algal cells are contained in the intestine (Fig. 4b, c). The photosynthetic efficiency $F_{\mathrm{v}} / F_{\mathrm{m}}>0.5$ determined for AEFW specimens indicates that their zooxanthellae are photosynthetically competent and might provide the parasites with nutritional benefits. Polyclad flatworms are known to be able to starve for months (Chintala and Kennedy 1993). Interestingly, isolated AEFWs without access to the host died after 5-7 d, suggesting that the algae do not represent a significant energy source for the animals but are most likely maintained solely for camouflage purposes. This remarkable camouflage strategy presumably represents an evolutionary adaptation of $A$. acroporae to the acroporid host. In this context, it is interesting to note that the visually similar camouflage of the Acropora-associated Waminoa representative may be the result of convergent evolution since acoels do not have branched guts (Hyman 1951).

Sequence analyses of the $18 \mathrm{~S}$ rDNA region demonstrated that the UK AEFW strain groups within the
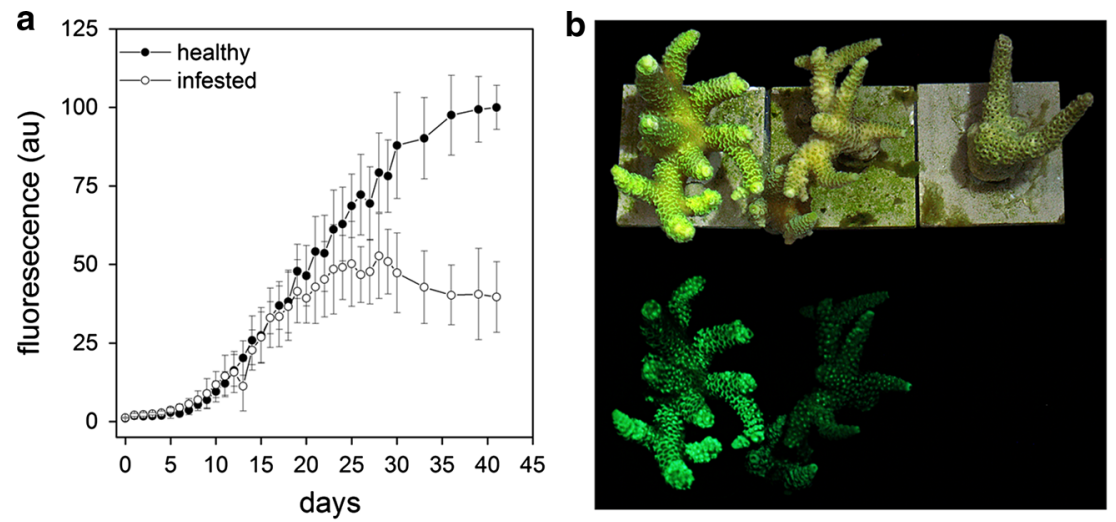

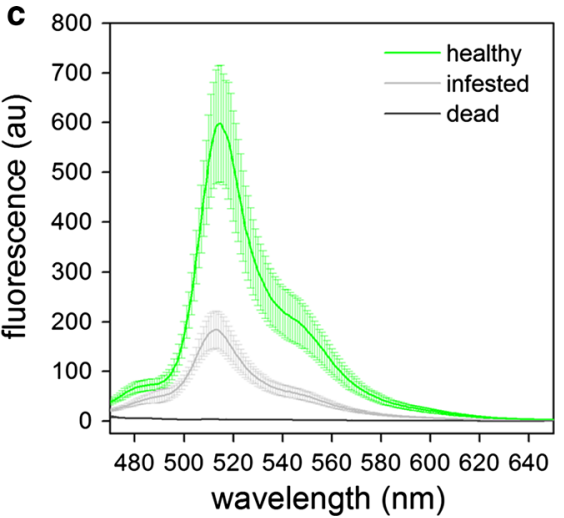

b Photographs of the daylight appearance (above) and the green fluorescence (below) of replicate colonies of a green colour morph of A. millepora during different stages of infestation ranging from healthy (left), over infested (middle) and to dead (right). c Quantitative comparison of the content of the GFP-like protein amilFP512 in the colonies depicted in $\mathbf{b}$ expressed as fluorescence emission per area (D'Angelo et al. 2008) 

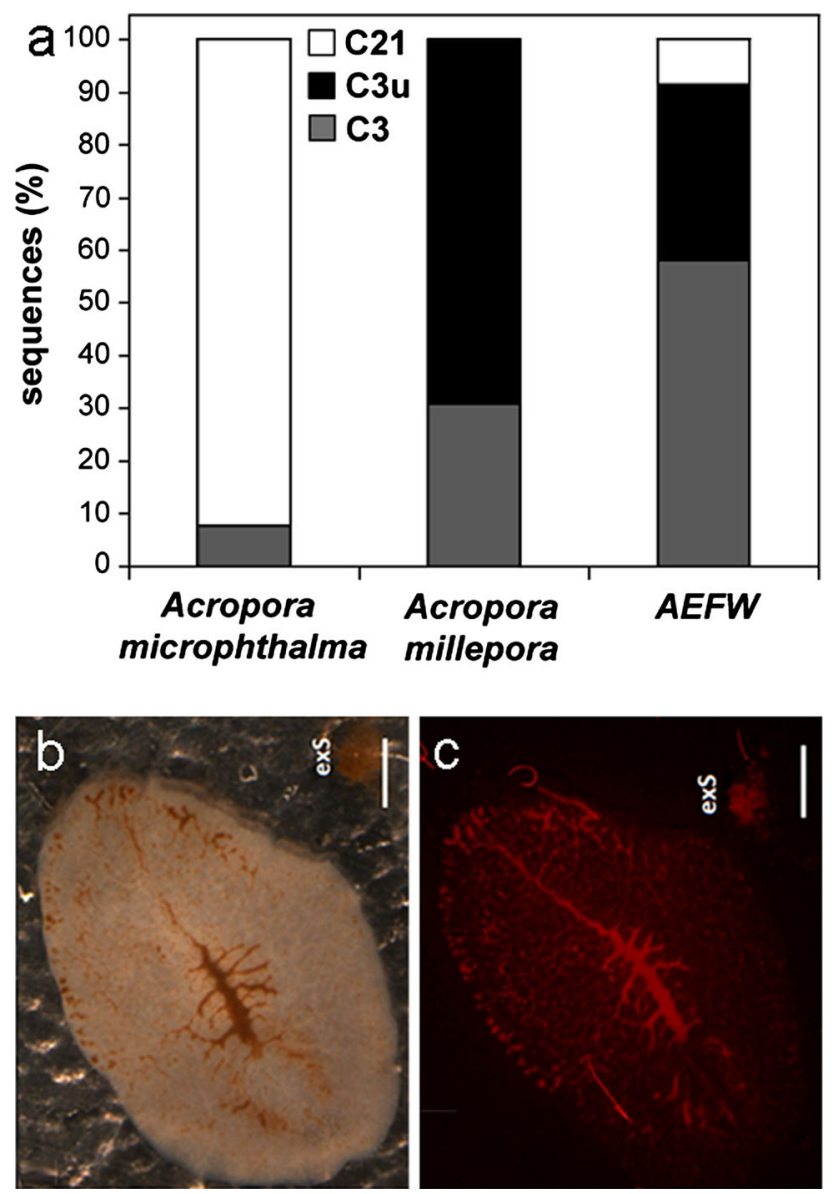

Fig. 4 Composition of the zooxanthellae (Symbiodinium spp.) community of an infested colony of A. millepora, of the associated AEFWs and of a co-cultured A. microphthalma colony. a Frequencies of subclades ( $\mathrm{C} 21, \mathrm{C} 3, \mathrm{Cu})$ were deduced from the detection frequency of the respective sequences among cloned ITS2 fragments. b, c Photographs of a heat-stressed AEFW after expulsion of the zooxanthellae. Images were acquired under white light (b) and in the fluorescence mode (c). Red chlorophyll fluorescence of zooxanthellae (c) was documented by the use of a TRITC filter set. Expelled Symbiodinium cells $($ exS $)$ are visible in the upper right corner of panels $\mathbf{b}, \mathbf{c}$

cotylean clade of polyclad flatworms (ESM Fig. 3a), ruling out the phenotypically similar Acropora-associated $\mathrm{Wa}$ minoa sp. from Okinawa (Matsushima et al. 2010). Analysis of the $28 \mathrm{~S}$ rDNA region identified the UK strain as the recently described A. acroporae, an AEFW from two US aquaria and from one natural location on Lizard Island (Rawlinson et al. 2011; Rawlinson and Stella 2012) (ESM Fig. 3b). Our $28 \mathrm{~S}$ rDNA sequence showed a $100 \%$ identity to the Virginia strain of $A$. acroporae, but could be distinguished in three shared residues from the New York and the Lizard Island strains. These nucleotide substitutions may indicate that at least two molecularly distinct A. acroporae strains are widely distributed in aquaria. Our global survey of reported AEFW infestations (Fig. 1, ESM Table 1) together with the results of the present paper revealed that many of the unidentified AEFWs show characteristics of $A$. acroporae such as exclusive preference for a diverse range of acroporids, excellent camouflage, preferred occurrence on the shaded branch sides, the typical 'bite marks' and egg clusters being deposited on dead skeletons (ESM Table 1). A. acroporae was considered responsible for the loss of Acropora colonies at Birch Aquarium (USA) (Nosratpour 2008; Rawlinson et al. 2011). Taken together, these data suggest that $A$. acroporae is globally distributed in land-based coral cultures, including regions close to natural coral reefs such as Florida, Thailand or Hong Kong (Fig. 1). We conclude that due to the broad range of accepted host coral species with a high preference for the cosmopolitan A. valida, the excellent camouflage and the ability to survive at least 5 days without host, A. acroporae has the potential to become a dangerous invasive species when released to an environment to which it is non-native. To better categorise this potential risk, further data on the biogeographic range of A. acroporae in reefs around the globe, on its natural predators and on its genetic diversity in captivity are urgently required.

Acknowledgments We thank the reviewers of this manuscript for their helpful suggestions. Funding: NERC (NE/K00641X/1 to JW; studentship to BCCH/JW), the European Union's Seventh Framework Programme (FP/2007-2013)/ERC Grant Agreement No. 311179 to JW.

Open Access This article is distributed under the terms of the Creative Commons Attribution License which permits any use, distribution, and reproduction in any medium, provided the original author(s) and the source are credited.

\section{References}

Barneah O, Brickner I, Hooge I, Weis VM, LaJeunesse TC, Benayahu Y (2007) Three party symbiosis: acoelomorph worms, corals and unicellular algal symbionts in Eilat (Red Sea). Mar Biol 151: $1215-1223$

Betancur-R R, Hines A, Acero A, Orti G, Wilbur AE, Freshwater DW (2011) Reconstructing the lionfish invasion: insights into Greater Caribbean biogeography. J Biogeogr 38:1281-1293

Chintala MM, Kennedy VS (1993) Reproduction of Stylochus ellipticus (Platyhelminthes: Polycladida) in response to temperature, food, and presence or absence of a partner. Biol Bull 185:373-387

D'Angelo C, Wiedenmann J (2012) An experimental mesocosm for long-term studies of reef corals. J Mar Biol Assoc UK 92: 769-775

D'Angelo C, Smith EG, Oswald F, Burt J, Tchernov D, Wiedenmann J (2012) Locally accelerated growth is part of the innate immune response and repair mechanisms in reef-building corals as detected by green fluorescent protein (GFP)-like pigments. Coral Reefs 31:1045-1056

D'Angelo C, Denzel A, Vogt A, Matz MV, Oswald F, Salih A, Nienhaus GU, Wiedenmann J (2008) Blue light regulation of 
host pigment in reef-building corals. Mar Ecol Prog Ser 364:97-106

Haapkylä J, Seymour AS, Barneah O, Brickner I, Hennige S, Suggett D, Smith D (2009) Association of Waminoa sp. (Acoela) with corals in the Wakatobi Marine Park, South-East Sulawesi, Indonesia. Mar Biol 156:1021-1027

Hughes TP, Rodrigues MJ, Bellwood DR, Ceccarelli D, HoeghGuldberg O, McCook L, Moltschaniwskyj N, Pratchett MS, Steneck RS, Willis B (2007) Phase shifts, herbivory, and the resilience of coral reefs to climate change. Curr Biol 17:360-365

Hume B, D'Angelo C, Burt J, Baker A, Riegl B, Wiedenmann J (2013) Corals from the Persian/Arabian Gulf as models for thermotolerant reef-builders: Prevalence of clade C3 Symbiodinium, host fluorescence and ex situ temperature tolerance. Mar Pollut Bull 72:313-322

Hyman LH (1951) The invertebrates: Platyhelminthes and Rhynchocoela: The acoelomate bilateria (Vol. 2). McGraw-Hill, New York

Leutenegger A, D’ Angelo C, Matz MV, Denzel A, Oswald F, Salih A, Nienhaus GU, Wiedenmann J (2007) It's cheap to be colorful. Anthozoans show a slow turnover of GFP-like proteins. FEBS J 274:2496-2505

Matsushima K, Fujiwara E, Hatta M (2010) An unidentified species of acoel flatworm in the genus Waminoa associated with the coral Acropora from the field in Japan. Galaxea 12:51

Naumann M, Mayr C, Struck U, Wild C (2010) Coral mucus stable isotope composition and labeling: experimental evidence for mucus uptake by epizoic acoelomorph worms. Mar Biol 157: 2521-2531

Nosratpour F (2008) Observations of a polyclad flatworm affecting acroporid corals in captivity. Advances in coral husbandry in public aquariums. Public Husbandry Series 2:37-46

Oswald F, Schmitt F, Leutenegger A, Ivanchenko S, D'Angelo C, Salih A, Maslakova S, Bulina M, Schirmbeck R, Nienhaus GU, Matz MV, Wiedenmann J (2007) Contributions of host and symbiont pigments to the coloration of reef corals. FEBS J 274:1102-1109
Padilla DK, Williams SL (2004) Beyond ballast water: aquarium and ornamental trades as sources of invasive species in aquatic ecosystems. Front Ecol Environ 2:131-138

Rawlinson KA, Stella JS (2012) Discovery of the corallivorous polyclad flatworm, Amakusaplana acroporae, on the Great Barrier Reef, Australia-the first report from the wild. PLoS ONE 7:e42240

Rawlinson KA, Gillis JA, Billings RE Jr, Borneman EH (2011) Taxonomy and life history of the Acropora-eating flatworm Amakusaplana acroporae nov. sp. (Polycladida: Prosthiostomidae). Coral Reefs 30:693-705

Rotjan RD, Lewis SM (2008) Impact of coral predators on tropical reefs. Mar Ecol Prog Ser 367:73-91

Savage AM, Goodson MS, Visram S, Trapido-Rosenthal H, Wiedenmann J, Douglas AE (2002) Molecular diversity of symbiotic algae at the latitudinal margins of their distribution: Dinoflagellates of the genus Symbiodinium in corals and sea anemones. Mar Ecol Prog Ser 244:17-26

Smith EG, D'Angelo C, Salih A, Wiedenmann J (2013) Screening by coral green fluorescent protein (GFP)-like chromoproteins supports a role in photoprotection of zooxanthellae. Coral Reefs 32:463-474

Tamura K, Peterson D, Peterson N, Stecher G, Nei M, Kumar S (2011) MEGA5: Molecular evolutionary genetics analysis using maximum likelihood, evolutionary distance, and maximum parsimony methods. Mol Biol Evol 28:2731-2739

Wiedenmann J, Baumstark A, Pillen TL, Meinesz A, Vogel W (2001) DNA fingerprints of Caulerpa taxifolia provide evidence for the introduction of an aquarium strain into the Mediterranean Sea and its close relationship to an Australian population. Mar Biol 138:229-234

Wiedenmann J, D'Angelo C, Smith EG, Hunt AN, Legiret F-E, Postle AD, Achterberg EP (2013) Nutrient enrichment can increase the susceptibility of reef corals to bleaching. Nat Clim Chang 3: 160-164 